\title{
Metabolic and Angiogenic Consequences of the Presence or Absence of UCP1
}

\author{
Barbara Cannon and Jan Nedergaard
}

\begin{abstract}
Adaptive adrenergic thermogenesis - both the form that develops subsequent to cold acclimation and the form that develops subsequent to a palatable diet challenge - is entirely dependent on the presence and activity of the brown fat uncoupling protein, UCP1. In a cold environment, the absence of UCP1 can be compensated by alternative means, such as shivering or exercise. Upon a challenge with a palatable diet, similar alternatives are not available, and mice become obese in the absence of UCP1. The recent identification of active brown fat in adult humans raises questions as to its role in protection from obesity and in a potential therapeutic context.
\end{abstract}

\section{Introduction}

It has been recognized for some 45 years that brown adipose tissue has a function as a heat-generating tissue. This finding was first described for animals arousing from hibernation and, subsequently, in small, cold-acclimated rodents and in mammalian neonates. The mechanism of thermogenesis was found to be through a mitochondrial uncoupling mediated by the prototype uncoupling protein, UCP1 (for review, see Cannon and Nedergaard 2004). However, the significance of the heat production was questioned for many years. After the generation of a mouse with an ablation in the UCP1 gene (Enerbäck et al. 1997), it became possible to evaluate its significance in a number of physiological and experimental situations. More recently, a role for brown fat thermogenesis was proposed in regulation of energy expenditure in connection with body weight maintenance (for review, see Cannon and Nedergaard 2004). This role has now also been evaluated in the UCP1-ablated mouse. That thermogenesis deriving from brown adipose tissue has a role in

\section{B. Cannon ( $\square)$}

The Wenner-Gren Institute, Stockholm University, Stockholm, Sweden

e-mail: barbara.cannon@wgi.su.se 
survival in cold and in body weight regulation became clear through these studies. However, these aspects concerning rodents can be considered esoteric and primarily of experimental interest. Therefore, it is of major importance that active brown adipose tissue has recently been identified in adult humans (Nedergaard et al. 2007; Cypess et al. 2009; Saito et al. 2009; van Marken Lichtenbelt et al. 2009; Virtanen et al. 2009; Zingaretti et al. 2009), and it now seems relevant to apply with caution the accumulated knowledge from animal experimentation.

\section{In the Absence of UCP1, Brown Fat Cells Do Not Produce Heat}

The absence of UCP1 does not negatively influence the growth and development of brown adipose tissue. It is therefore possible to isolate mature brown adipocytes from tissue from wild-type and UCP1-ablated mice and compare their thermogenic responsiveness to the sympathetic neurotransmitter norepinephrine. Cells from UCP1-ablated mice lack the ability to elevate heat production in response to noradrenergic stimulation, demonstrating that indeed all regulated brown adipocyte heat production is mediated via this endogenous mitochondrial uncoupler (Fig. 1; Matthias et al. 2000). It is also noteworthy that, prior to stimulation by norepinephrine, the basal respiration of both types of cells is identical. Thus, UCP1 does not possess any inherent leakiness, and therefore its presence without simultaneous activation will not influence an animal's metabolism. While this fact can be clearly observed in isolated brown adipocytes, it has not been generally recognized with respect to whole animal metabolism. However, if wild-type animals with no UCP1 are compared to animals with high UCP1 levels, the basal metabolism of the animals is identical. Endogenously expressed UCP1 only influences metabolism if it is activated.

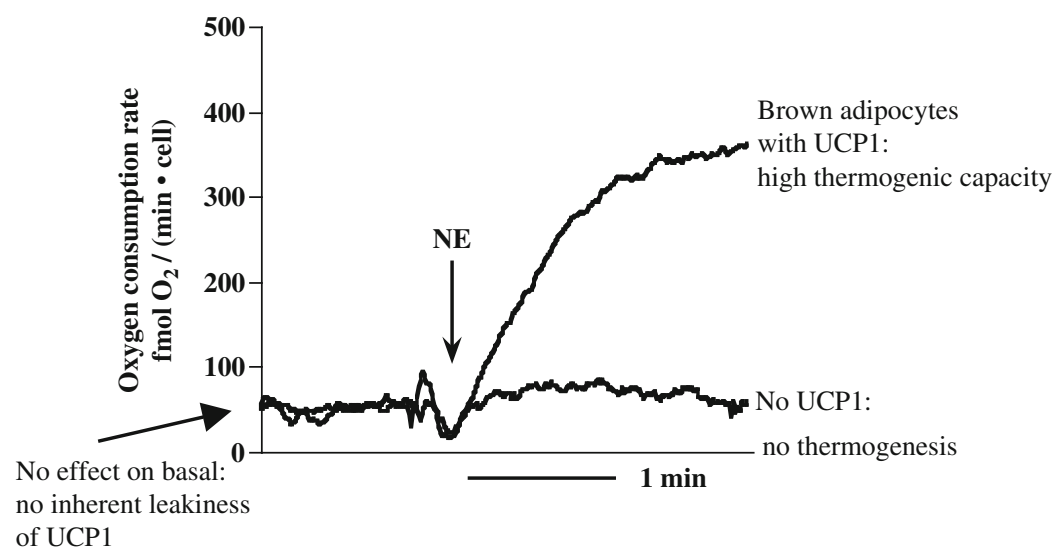

Fig. 1 UCP1 is essential for norepinephrine-induced thermogenesis in brown adipocytes. NE: norepinephrine. Adapted from Matthias et al. 2000 


\section{Does the Presence/Absence of UCP1 Affect Adaptive Nonshivering Thermogenesis?}

When a mammal is exposed acutely to a cold environment, it will attempt to defend its body temperature by elevating its heat production through shivering. For a mouse, this response can result in an increased metabolism of some 4- to 5-fold over basal level, with a consequent parallel increase in food intake. In the 1950s, a remarkable phenomenon was observed: if the animal remained in a cold environment for some weeks, the shivering ceased but the metabolism remained equally high. The heat production that was occurring in the absence of shivering became known as nonshivering thermogenesis. The site of this heat production was discussed as being muscle or, somewhat later, possibly brown adipose tissue. Elegant studies in the late 1970s should have resolved this question, when it was shown that blood flow to muscle was not increased whereas blood flow to brown adipose tissue was massively increased in cold-acclimated animals, but this finding was not generally accepted as proof for brown fat's exclusive role (Foster and Frydman 1979). The UCP1-ablated mouse allowed clarification of the question, when it became evident that these animals could survive a prolonged period in cold in just the same way as acutely cold-exposed animals by using constant muscle shivering (Golozoubova et al. 2001). Thus, there is no cold acclimation-induced nonshivering thermogenesis without UCP1.

These experiments also demonstrated that animals can survive in cold in the absence of UCP1, provided that they have sufficient power and endurance in their muscles to maintain constant shivering at a level that elevates metabolism 5-fold over basal. There would seem to be two advantages to having the ability to develop nonshivering thermogenesis in a cold environment: firstly that it is considerably more comfortable, and secondly that, as long as the heart and lungs still have excess capacity, the animal can, in a new cold emergency, once again switch on its muscle shivering over and above the metabolic level reached by the nonshivering heat production, providing a survival advantage.

\section{Does the Presence/Absence of UCP1 Affect Adaptive Adrenergic Thermogenesis?}

Sympathetic activation of heat production can be mimicked by injection of a high dose of norepinephrine into an animal, flooding the adrenergic receptors of the body and in this way activating all adrenergic processes, even those normally controlled by activation of the sympathetic nervous system. Injection of norepinephrine into a mouse that has been housed at its thermoneutral temperature of $30^{\circ} \mathrm{C}$ - that is, the temperature where it does not have to expend extra energy to elevate metabolism to defend body temperature (i.e., demonstrates its basal metabolism) - results in a modest increase in oxygen consumption (thermogenesis). A similar injection into 

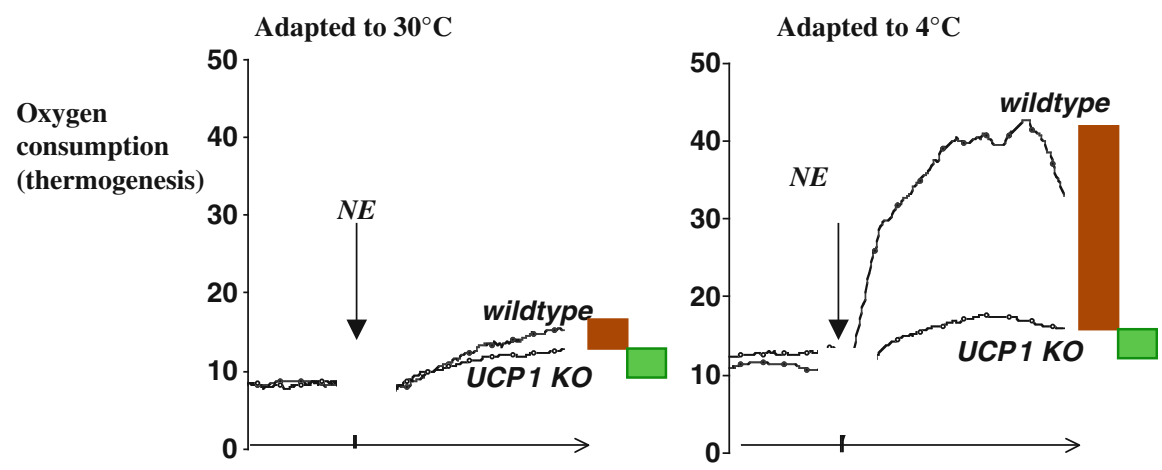

Fig. 2 No cold-recruited adaptive adrenergic nonshivering thermogenesis without UCP1. Anesthetized intact mice were injected with norepinephrine (NE) at $30^{\circ} \mathrm{C}$. Green bars indicate the UCP1-independent response to NE, brown bars the UCP1-dependent response. Clearly the former is not adaptive whereas the latter is. Golozoubova, V., unpublished

an animal that has been housed for some weeks at $5^{\circ} \mathrm{C}$ results in a 4- to 5-fold higher increase, a magnitude very similar to that level of metabolism maintained by the animal at the temperature of acclimation. This increase in metabolic responsiveness is termed adaptive adrenergic thermogenesis.

When norepinephrine is injected into a UCP1-ablated mouse that has been kept at thermoneutrality, the metabolic response is similar to but somewhat lower than that seen in the wild-type mouse. After the UCP1-ablated mouse has been acclimated to $5^{\circ} \mathrm{C}$, the norepinephrine response is unchanged from that shown in the mouse acclimated at $30^{\circ} \mathrm{C}$ (Fig. 2; Golozoubova et al. 2006). This finding provides information on two points: 1) at $30^{\circ} \mathrm{C}$, mice have a small, UCP1-dependent metabolic capacity, the function of which may be related to energy expenditure in a dietary connection (see below), and 2) the increase in adrenergic responsiveness resulting from cold acclimation is entirely dependent upon UCP1.

\section{Does the Presence/Absence of UCP1 Affect Cold-Induced Angiogenesis?}

When an animal acclimates to a cold environment, the brown adipose tissue grows through cell proliferation and differentiation. This tissue growth explains the relatively slow acclimation process (weeks). However, in addition to adipocyte development, an increase in the vasculature is an absolute requirement, not least in consideration of the marked increase in metabolism that occurs and the increased blood flow referred to above. Neoangiogenesis is often believed to occur as a result of developing hypoxia in a growing tissue. Indeed, in brown adipose tissue of coldacclimating animals, hypoxia can be demonstrated. However, in UCP1-ablated mice, where no hypoxia can develop because metabolism cannot be elevated 
without UCP1, neo-angiogenesis in the cold occurs normally (Xue et al. 2009). Thus, the angiogenesis results from the adrenergic stimulation of the tissue and is not dependent on a hypoxic environment, and thus not on the presence or activity of UCP1.

\section{Does the Presence/Absence of UCP1 Affect $\boldsymbol{\beta}_{3}$-Adrenoceptor- Induced Thermogenesis and Weight Loss?}

Of the $\beta$-adrenergic receptor subtypes, the $\beta_{3}$-subtype is that found predominantly in rodent adipose tissues. It is through this subtype that thermogenesis in brown adipocytes is mediated. In non-cold-acclimated mice, injection of a $\beta_{3}$-selective adrenergic agonist elicits an approximately $30 \%$ increase in metabolism over basal level. In UCP1-ablated animals, no such increase is observed (Inokuma et al. 2006).

Prolonged treatment with a selective $\beta_{3}$-agonist stabilizes body weight in wildtype mice fed a cafeteria diet, whereas in UCP1-ablated mice, body weight continues to increase in spite of the $\beta_{3}$-agonist treatment (Inokuma et al. 2006). Thus, in the absence of UCP1, effects of $\beta_{3}$-agonists on metabolism and body weight control are lost.

\section{Does the Presence/Absence of UCP1 Affect Diet-Induced Thermogenesis?}

Diet-induced thermogenesis is used in this context to mean diet adaptationrecruited, norepinephrine-induced thermogenesis. It is that increase in responsiveness to an injection of norepinephrine that is observed in mice fed a palatable diet compared to those fed a control diet.

As stated above, wild-type mice housed at thermoneutrality have a marginally larger increase in norepinephrine responsiveness compared to UCP1-ablated mice, indicating that a small amount of UCP1 is present in the wild-type mice, not for thermogenic purposes but for another purpose, possibly related to diet-induced thermogenesis. The difference in response between the UCP1-ablated mice and the wild-type mice is thus a UCP1-dependent thermogenesis.

After being fed a high-fat diet, wild-type mice generally show an approximately twice as large total response to norepinephrine as control-fed animals. The high fatfed, UCP1-ablated animals fail to increase their response above that seen in the control-fed animals. Subtraction of the response in the UCP1-ablated animals from that seen in the high fat-fed wild-type animals gives a measure of the UCP1dependent diet-induced adrenergic thermogenesis. It is approximately 4-fold larger than for the control-fed animals. As said, no increase in the magnitude of the adrenergic thermogenesis is seen following high-fat diet feeding in the UCP1-ablated 

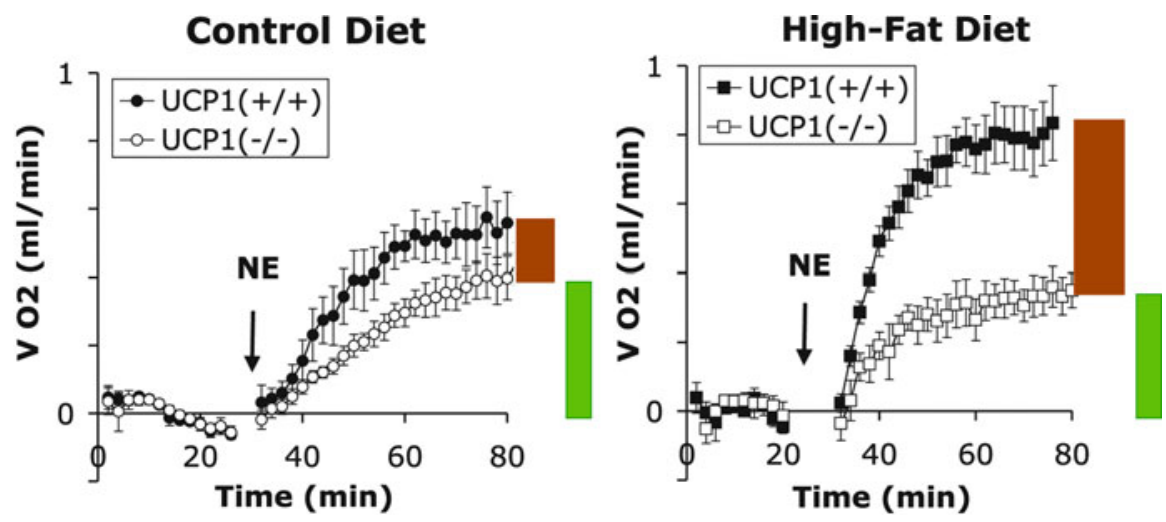

Fig. 3 No diet-recruited adaptive adrenergic nonshivering thermogenesis without UCP1. Green bars indicate the UCP1-independent response to NE, brown bars the UCP1-dependent response. Clearly the former is not adaptive whereas the latter is. Adapted from Feldmann et al. 2009

mice. This response, which is thus non-adaptive, is presumably the pharmacological response to a high dose of norepinephrine, as mentioned above. The diet adaptationrecruited, norepinephrine-induced thermogenesis is, however, completely dependent on the presence of UCP1, just as is the cold-recruited, adaptive adrenergic thermogenesis (Fig. 3; Feldmann et al. 2009).

\section{Does the Presence/Absence of UCP1 Affect Leptin-Induced Thermogenesis?}

The adipose tissue hormone leptin, acting at sites in the hypothalamus, can modulate body weight and body energy stores in two ways: by reducing food intake and by enhancing thermogenesis. To study its effect on body weight resulting from an effect on energy expenditure, control animals must be pair-fed to leptin-treated animals to adjust to the decrease in food intake induced by leptin, with the concomitant reduction in body weight. When this is done, a further modest decrease in body weight is seen in leptin-treated wild-type mice. In UCP1-ablated mice, no further decrease in body weight is seen (Commins et al. 2001).

When mice are food-restricted, they reduce energy expenditure (oxygen consumption) in an attempt to conserve energy. Injection of wild-type mice with leptin prevents this conservation strategy, and the wild-type mice maintain their unrestricted oxygen consumption. UCP1-ablated mice also reduce oxygen consumption upon food restriction, but leptin injection is subsequently without effect on preventing the decrease (Okamatsu-Ogura et al. 2007). Thus, without UCP1, there is no leptin-induced thermogenesis. 


\section{What are the Consequences of a Lack of Diet-Induced Thermogenesis?}

In the absence of UCP1, animals in a cold environment have alternative means (shivering, exercise) by which to defend body temperature. On a palatable diet at unchanged environmental temperature, animals lack such options to compensate for any loss of UCP1-mediated thermogenesis. Consequently, if brown fat thermogenesis plays a role in body weight regulation, animals without UCP1 should increase in body weight, particularly when fed a palatable diet. In initial reports on these mice, no such effect was reported. However, if animals are maintained at thermoneutrality, at a temperature in which they do not need heat from any source to maintain body temperature, mice increase in body weight and body fat content both on a control diet and, more markedly, on a high-fat diet (Fig. 4; Feldmann et al. 2009).
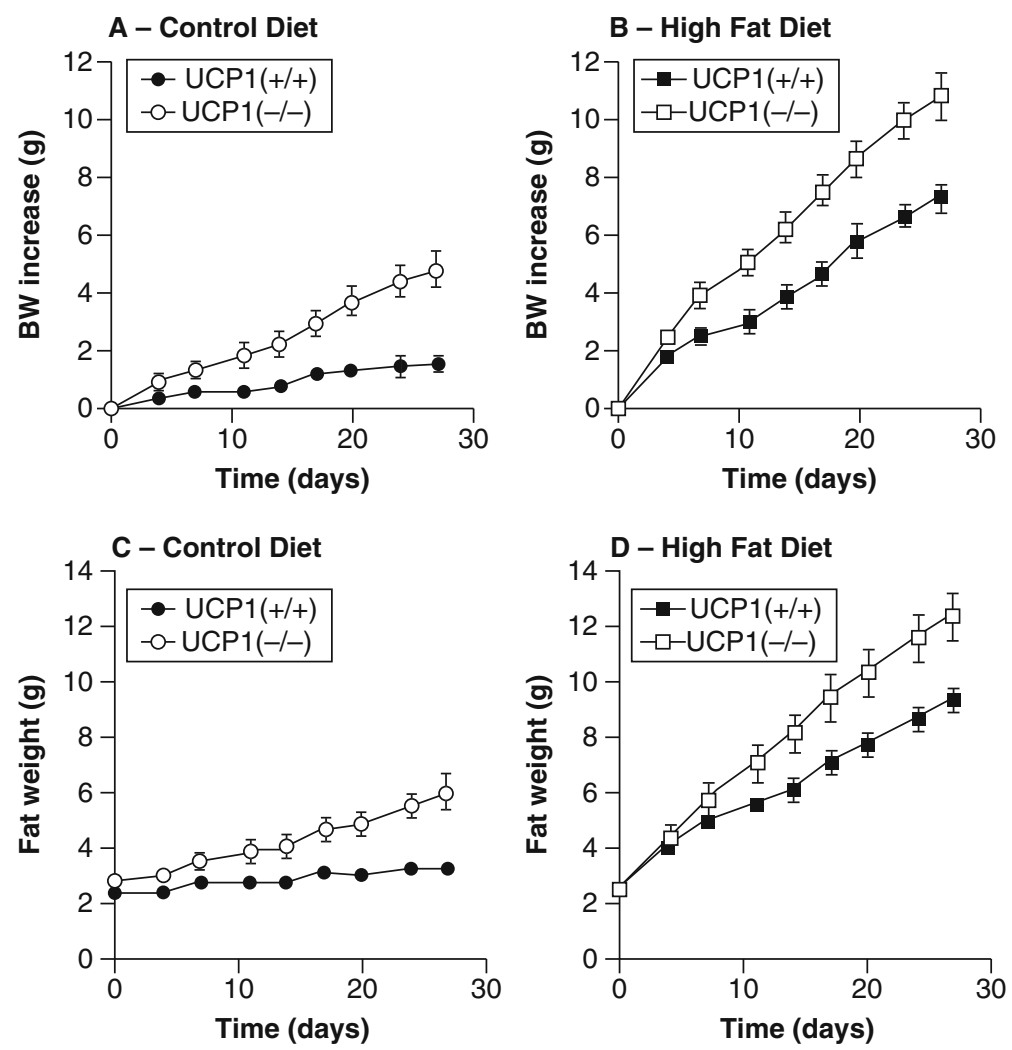

Fig. 4 Without brown fat, mice become fatter. Modified from a supplementary figure in Feldmann et al. 2009. BW: body weight 
These studies were performed on mice of the C57/B16 strain, a notoriously obesogenic strain. Mice of the $129 \mathrm{~Sv}$ strain are known to be obesity resistant and it is therefore of interest to observe the responses of these mice to both the absence of UCP1 and a dietary challenge. In $129 \mathrm{~Sv}$ wild-type mice maintained at thermoneutrality, a high-fat diet does not augment the rate of body weight increase over that seen when mice are fed a control diet. Thus these mice are protected from highfat diet-induced obesity. On a control diet, UCP1-ablated mice of the 129Sv strain also maintain the same body weight growth curve as the wild-type mice, therefore not showing the control diet-induced obesity seen with the C57/B16 mice. However, when UCP1-ablated mice on a 129Sv-background are fed a high-fat diet, they do become obese. The absence of UCP1 in combination with a palatable diet therefore overcomes the obesity resistance of this strain.

To understand this phenomenon, food intake and metabolic efficiency have been determined in the $129 \mathrm{~Sv}$ mice of both phenotypes. On the control diet, the UCP1ablated mice actually eat less than the wild-type mice. This small but significant decrease in food intake is adequate to allow the animals to maintain the same body weight as the wild-type animals that possess UCP1-mediated thermogenesis. On a high-fat diet, this moderation in food intake by the UCP1-ablated mice is lost, and both groups of animals eat the same amount of energy, but the UCP1-ablated mice now become obese.

The following conclusions can be drawn. On a control diet, UCP1-ablated mice that maintain the same growth curve as wild-type mice have a somewhat higher metabolic efficiency (body weight increase per food intake) than the wild-type mice, in that they eat less yet maintain the same growth rate. When the wild-type mice are challenged with a high-fat diet, they decrease their metabolic efficiency. The UCP1-ablated mice fed the high-fat diet are unable to decrease their metabolic efficiency. They therefore increase in body weight more than do the wild-type animals (Feldmann et al., submitted for publication). Thus, in the absence of UCP1, a lowered efficiency cannot be induced.

\section{Does the Presence/Absence of UCP1 Affect Human Metabolism?}

Although brown adipose tissue has been identified in adult humans (Nedergaard et al. 2007; Cypess et al. 2009; Saito et al. 2009; van Marken Lichtenbelt et al. 2009; Virtanen et al. 2009; Zingaretti et al. 2009), it is too early to conclude to what degree the presence or absence of UCP1 and active brown adipose tissue impact upon body weight. However, there are certain inferences from the literature that can be said to support the data obtained in mice. There is a polymorphism in the distal enhancer of the human UCP1 gene (-3826), and possession of the AA alleles is associated with a higher expression of UCP1 than is possession of the GG alleles (Esterbauer et al. 1998). Furthermore, a higher percentage of AA allele carriers are found in the lowest quartile of body mass index in a representative 
population, whereas the GG alleles are overrepresented in the heaviest quartile (Clement et al. 1996; Sramkova et al. 2007).

Immunohistochemical analysis of samples of brown fat from the neck region of patients undergoing thyroid surgery demonstated that the tissue is verified brown fat, because of a rich presence of UCP1 staining in islets of multilocular adipocytes. Correlation of UCP1-containing samples with body mass index indicates that the leaner a person, the higher the likelihood of possessing UCP1. However, the same correlation was observed as a function of age; the younger a patient, the greater the likelihood of possessing UCP1 (Zingaretti et al. 2009). Thus, young and lean persons have a higher likelihood of possessing UCP1 and active brown adipose tissue than old and obese persons.

While it is not yet appropriate to conclude that obesity depends upon a decrease in UCP1 activity, it is possible, based on the animal data, to infer that there may be an influence of UCP1 on adult human metabolism.

Another conclusion that may be drawn is that therapeutic measures based on brown fat thermogenic activity need to be very carefully designed. People may be obese and nonetheless be in possession of some active brown fat and UCP1. This finding would be in accord with wild-type animals fed a palatable diet, in which UCP1 content increases so that metabolic efficiency decreases, leading to animals that become moderately more obese but not as obese as they should become were they to have increased food intake and an unchanged efficiency. Thus, brown fat activity has been beneficial but has not managed fully to compensate for the excess food intake. Equivalent animals that lack UCP1 and can therefore not decrease metabolic efficiency become progressively more obese than their wildtype counterparts.

In a human scenario, it would be appropriate to treat patients with an agent such as a $\beta_{3}$-agonist only if it could be shown that they already possessed measurable brown fat. While it may be speculated that a majority of adult humans probably possess progenitor cells for brown adipocytes, it would only be feasible to treat such patients with a $\beta_{3}$-agonist if they had been previously treated with an agent to increase the content of brown adipocytes. Since a safe means to increase the cell proliferation is still lacking, widespread use of brown adipose tissue thermogenesis for treatment of human obesity must await development of such suitable agents.

\section{References}

Cannon B, Nedergaard J (2004) Brown adipose tissue: function and physiological significance. Physiol Rev 84:277-359

Clement K, Ruiz J, Cassard-Doulcier AM, Bouillaud F, Ricquier D, Basdevant A, Guy-Grand B, Froguel P (1996) Additive effect of A-->G (-3826) variant of the uncoupling protein gene and the Trp64Arg mutation of the beta 3 -adrenergic receptor gene on weight gain in morbid obesity. Int J Obes 20:1062-1066

Commins SP, Watson PM, Frampton IC, Gettys TW (2001) Leptin selectively reduces white adipose tissue in mice via a UCP1-dependent mechanism in brown adipose tissue. Am J Physiol 280:E372-E373 
Cypess AM, Lehman S, Williams G, Tal I, Rodman D, Goldfine AB, Kuo FC, Palmer EL, Tseng Y-H, Doria A, Kolodny GM, Kahn CR (2009) Identification and importance of brown adipose tissue in adult humans. New Engl J Med 360:1509-1517

Enerbäck S, Jacobsson A, Simpson EM, Guerra C, Yamashita H, Harper M-E, Kozak LP (1997) Mice lacking mitochondrial uncoupling protein are cold-sensitive but not obese. Nature 387:90-94

Esterbauer H, Oberkofler H, Liu Y, Breban D, Hell E, Krempler F, Patsch W (1998) Uncoupling protein-1 mRNA expression in obese human subjects: the role of sequence variations at the uncoupling protein-1 gene locus. J Lipid Res 39:834-844

Feldmann HM, Golozoubova V, Cannon B, Nedergaard J (2009) UCP1 ablation induces obesity and abolishes diet-induced thermogenesis in mice exempt from thermal stress by living at thermoneutrality. Cell Metab 9:203-209

Foster DO, Frydman ML (1979) Tissue distribution of cold-induced thermogenesis in conscious warm- or cold-acclimated rats reevaluated from changes in tissue blood flow: the dominant role of brown adipose tissue in the replacement of shivering by nonshivering thermogenesis. Can J Physiol Pharmacol 57:257-270

Golozoubova V, Cannon B, Nedergaard J (2006) UCP1 is essential for adaptive adrenergic nonshivering thermogenesis. Am J Physiol 291:E350-E357

Golozoubova V, Hohtola E, Matthias A, Jacobsson A, Cannon B, Nedergaard J (2001) Only UCP1 can mediate adaptive nonshivering thermogenesis in the cold. FASEB J 15:2048-2050

Granneman JG, Burnazi M, Zhu Z, Schwamb LA (2003) White adipose tissue contributes to UCP1-independent thermogenesis. Am J Physiol Endocrinol Metab 285:E1230-E1236

Inokuma K-i, Okamatsu-Ogura Y, Omachi A, Matsushita Y, Kimura K, Yamashita H, Saito M (2006) Indispensable role of mitochondrial UCP1 for antiobesity effect of beta3-adrenergic stimulation. Am J Physiol 290:E1014-E1021

Matthias A, Ohlson KEB, Fredriksson JM, Jacobsson A, Nedergaard J, Cannon B (2000) Thermogenic responses in brown-fat cells are fully UCP1-dependent: UCP2 or UCP3 do not substitute for UCP1 in adrenergically or fatty-acid induced thermogenesis. J Biol Chem 275:25073-25081

Nedergaard J, Bengtsson T, Cannon B (2007) Unexpected evidence for active brown adipose tissue in adult humans. Am J Physiol 293:E444-E452

Okamatsu-Ogura Y, Uozumi A, Toda C, Kimura K, Yamashita H, Saito M (2007) Uncoupling protein 1 contributes to fat-reducing effect of leptin. Obes Res Clin Pract 1:233-241

Saito M, Okamatsu-Ogura Y, Matsushita M, Watanabe K, Yoneshiro T, Nio-Kobayashi J, Iwanaga T, Miyagawa M, Kameya T, Nakada K, Kawai Y, Tsujisaki M (2009) High incidence of metabolically active brown adipose tissue in healthy adult humans: effects of cold exposure and adiposity. Diabetes 58:1526-1531

Sramkova D, Krejbichova S, Vcelak J, Vankova M, Samalikova P, Hill M, Kvasnickova H, Dvorakova K, Vondra K, Hainer V, Bendlova B (2007) The UCP1 gene polymorphism A3826G in relation to DM2 and body composition in Czech population. Exp Clin Endocrinol Diabetes 115:303-307

van Marken Lichtenbelt WD, Vanhommerig JW, Smulders NM, Drossaerts JM, Kemerink GJ, Bouvy ND, Schrauwen P, Teule GJ (2009) Cold-activated brown adipose tissue in healthy men. New Engl J Med 360:1500-1508

Virtanen KA, Lidell ME, Orava J, Heglind M, Westergren R, Niemi T, Taittonen M, Laine J, Savisto NJ, Enerbäck S, Nuutila P (2009) Functional brown adipose tissue in healthy adults. New Engl J Med 360:1518-1525

Xue Y, Petrovic N, Cao R, Larsson O, Lim S, Chen S, Feldmann HM, Liang Z, Zhu Z, Nedergaard J, Cannon B, Cao Y (2009) Hypoxia-independent angiogenesis in adipose tissues during cold acclimation. Cell Metab 7:99-109

Zingaretti MC, Crosta F, Vitali A, Guerrieri M, Frontini A, Cannon B, Nedergaard J, Cinti S (2009) The presence of UCP1 demonstrates that metabolically active adipose tissue in the neck of adult humans truly represents brown adipose tissue. FASEB J 23:3113-3120 